\title{
Asymptotic expansions for approximate eigenvalues of integral operators with a weakly singular periodic kernel
}

\author{
Rekha P. Kulkarni and Akshay S. Rane* \\ Department of Mathematics, \\ Indian Institute of Technology Bombay, \\ Powai, Mumbai 400 076, India \\ Email: rpk@math.iitb.ac.in \\ Email: akshayrane11@gmail.com \\ *Corresponding author
}

\begin{abstract}
We consider the approximation of an eigenvalue of an integral operator with a weakly singular periodic kernel using the modified Nyström scheme based on the composite trapezoidal rule. Numerical results are presented which confirm the theoretical orders of convergence.
\end{abstract}

Keywords: asymptotic expansion; modified Nyström scheme; weakly singular periodic kernel.

Reference to this paper should be made as follows: Kulkarni, R.P. and Rane, A.S. (2016) 'Asymptotic expansions for approximate eigenvalues of integral operators with a weakly singular periodic kernel', Int. J. Convergence Computing, Vol. 2, No. 1, pp.79-92.

Biographical notes: Rekha P. Kulkarni is a Professor at the Indian Institute of Technology Bombay, Mumbai. Her broad area of research is numerical functional analysis.

Akshay S. Rane is an Assistant Professor at Institute of Chemical Technology, Mumbai. His broad area of research is numerical functional analysis.

This paper is a revised and expanded version of a paper entitled 'Asymptotic expansions for approximate eigenvalues of integral operators with weakly singular periodic kernel' presented at International Conference on Mathematical and Computational Sciences, Kannur, Kerela, 22-24 January 2015.

\section{Introduction}

Eigenvalue problems occur in many physical and engineering applications. They have been studied extensively in the research literature. One way of improving the orders of convergence of the approximate eigenelements is to establish asymptotic expansion for the eigenelements and then use Richardson extrapolation. Asymptotic expansions of approximate eigenvalues of integral operators with smooth kernels have been studied by Baker (1977) and Kulkarni (1997). Asymptotic expansions of approximate eigenvalues of 
integral operators with Green's kernel have been studied in Baker (1977), Kulkarni and Rane (2012). In this article, we consider the following integral operator

$$
(T x)(s)=\int_{a}^{b} k(s, t) x(t) d t, \quad s \in[a, b],
$$

with a weakly singular periodic kernel of the form

$$
k(s, t)=H_{1}(s, t) \log |s-t|+H_{2}(s, t) .
$$

1 We assume that $k(\cdot, \cdot)$ is periodic in both variables with period $Q=b-a$, and that for an even positive integer $m, H_{1}, H_{2} \in C^{m+2}(\{(s, t):|\mathbf{s}-\mathrm{t}| \leq 3 Q / 2, t \in \mathbb{R}\})$, the space of $m+2$ times continuously differentiable complex valued functions.

2 In addition, we also assume that $H_{1}(s, s)$ and $H_{2}(s, s)$ are periodic with period $Q$.

An example of such a kernel (see Sidi, 1989) is

$$
k(s, t)=\log \left(4 \sin \frac{|s-t|}{2}\right), \quad s, t \in[0,2 \pi] .
$$

The above kernel $k(s, t)$ can be written as

$$
k(s, t)=H_{1}(s, t) \log |s-t|+H_{2}(s, t),
$$

where

$$
H_{1}(s, t)=1 \text { and } H_{2}(s, t)=\log \left(\frac{4 \sin \frac{|s-t|}{2}}{|s-t|}\right) .
$$

Moreover $H_{1}(s, s)=1$ and $H_{2}(s, s)=\log 2$. Clearly the kernel $k(s, t)$ is $2 \pi$ periodic in both the variables. $H_{1}(s, s)=1$ and $H_{2}(s, s)=\log 2$ are constant functions so that they are periodic of any period and hence $2 \pi$ periodic. Thus assumptions 1 and 2 in the introduction about the kernel are trivially satisfied. Let $C_{Q}(\mathbb{R})$ be the space of all continuous functions on $\mathbb{R}$ that are periodic with period $Q=b-a$. Then $T: C_{Q}(\mathbb{R}) \rightarrow C_{Q}(\mathbb{R})$ is a compact operator (see Xu and Zhao, 1996). Since $T$ is a compact, the spectrum of $T, \sigma(T)$, which is the complement of the resolvent $\rho(T)=\left\{z \in \mathbb{C}:(T-z I)^{-1}: C_{Q}(\mathbb{R}) \rightarrow C_{Q}(\mathbb{R})\right.$ bounded linear $\}$, forms a countably infinite set with possibly 0 as the only limit point. Moreover, the non-zero elements of $\sigma(T)$ are eigenvalues with finite algebraic multiplicities. We are interested in an approximate solution of the eigenvalue problem

$$
T \varphi=\lambda \varphi, 0 \neq \lambda \in \mathbb{C}, 0 \neq \varphi \in C_{Q}(\mathbb{R}) .
$$

$\mathrm{Xu}$ and Xhao (1996) have considered the case of an approximate solution of the operator equation. They define a modified Nyström scheme $T_{n}$, which is a collectively compact family of operators converging to $T$ pointwise. An asymptotic expansion for $T_{n}$ is obtained and then an asymptotic expansion for the approximate solution of an operator equation is studied. The main purpose of this article is to obtain an asymptotic expansion for a simple eigenvalue $\lambda_{n}$. 
Since, for a fixed $t \in[a, b], k(\cdot, t)$ is periodic with period $Q$, we obtain

$$
\begin{aligned}
\lambda \varphi(s+Q) & =\int_{a}^{b} k(s+Q, t) \varphi(t) d t \\
& =\int_{a}^{b} k(s, t) \varphi(t) d t \\
& =\lambda \varphi(s), \quad s \in[a, b] .
\end{aligned}
$$

Since $\lambda \neq 0$, it follows that

$$
\varphi(s+Q)=\varphi(s), \quad s \in[a, b]
$$

This shows that the eigenfunction $\varphi$ is periodic with period $Q$.

In general, the eigenfunction $\varphi=C^{m+2}(a, b)$, but the derivatives of $\varphi$ may be unbounded at $a$ or $b$ (see Richter, 1976; Schneider, 1979). However, we show that in this particular case, $\varphi \in C^{m+2}(\mathbb{R})$.

Since $k$ is periodic in both variables and $x \in C_{Q}(\mathbb{R})$, we can write

$$
(T x)(s)=\int_{a}^{b} k(s, t) x(t) d t=\int_{a^{\prime}}^{b^{\prime}} k(s, t) x(t) d t,
$$

where $b^{\prime}-a^{\prime}=b-a=Q$. Choose $a^{\prime}$ and $b^{\prime}$ such that $b^{\prime}-a^{\prime}=b-a=Q$ and $a \in\left(a^{\prime}, b^{\prime}\right)$. Then since

$$
(T \varphi)(s)=\int_{a^{\prime}}^{b^{\prime}} k(s, t) \varphi(t) d t=\lambda \varphi(s), \quad s \in\left[a^{\prime}, b^{\prime}\right],
$$

it follows that $\varphi=C^{m+2}\left(a^{\prime}, b^{\prime}\right)$ and hence, in particular, $\varphi$ is $m+2$ times continuously differentiable at $a$. In a similar manner, it can be shown that $\varphi$ is $m+2$ times continuously differentiable at $b$. In fact, $\varphi \in C^{m+2}(\mathbb{R})$.

\section{Generalised Euler-MacLaurin formula and Nyström scheme}

Let $n \in \mathbb{N}$ and $h=\frac{b-a}{n}$. Let

$$
t_{i}=a+(i-1) h, \quad i=1, \ldots, n+1 .
$$

Then $a=t_{1}<t_{2}<\ldots<t_{n+1}=b$, is a uniform partition of $[a, b]$. We quote a result about the Euler-MacLaurin summation formula for a function of the form

$$
G(t)=g_{1}(t) \log |s-t|+g_{2}(t), \quad t \in\left[a-\frac{Q}{2}, b+\frac{Q}{2}\right], s \in[a, b],
$$

where $g_{1}, g_{2} \in C^{m+2}\left[a-\frac{Q}{2}, b+\frac{Q}{2}\right]$ (see Theorem 2.2 of Xu and Zhao, 1996).

Theorem 2.1: Assume that $G$ is periodic with period $Q=b-a$ on $\mathbb{R} \backslash\{s+k Q\}_{k=-\infty}^{\infty}$. Then 


$$
\begin{aligned}
\int_{a}^{b} G(t) d t= & h \sum_{j \neq 0, a<s+j h \leq b} G(s+j h)+g_{1}(s) h \log \left(\frac{h}{2 \pi}\right)+h g_{2}(s) \\
& +2 \sum_{\mu=1}^{m / 2} \frac{\zeta^{\prime}(-2 \mu)}{(2 \mu) !} g_{1}^{2 \mu}(s) h^{2 \mu+1}+O\left(h^{m+2}\right),
\end{aligned}
$$

where $\zeta$ is the Riemann zeta function.

Let $T$ be the integral operator, with kernel defined by equation (2). Let $x \in C_{Q}^{m+2}(\mathbb{R})$, the space of $Q$ periodic functions in $C^{m+2}(\mathbb{R})$. For a fixed $s \in[a, b]$, applying Theorem 2.1 to $G(t)=k(s, t) x(t)=H_{1}(s, t) \log |s-t| x(t)+H_{2}(s, t) x(t)$, we obtain

$$
\begin{aligned}
(T x)(s)= & h \sum_{j \neq 0, a<s+j h \leq b} k(s, s+j h) x(s+j h)+H_{1}(s, s) x(s) h \log \left(\frac{h}{2 \pi}\right) \\
& +h H_{2}(s, s) x(s)+\left.2 \sum_{\mu=1}^{m / 2} \frac{\zeta^{\prime}(-2 \mu)}{(2 \mu) !}\left(\frac{\partial^{2 \mu}}{\partial t^{2 \mu}} H_{1}(s, t) x(t)\right)\right|_{t=s} h^{2 \mu+1} \\
& +O\left(h^{m+2}\right) . \\
= & \left(T_{n} x\right)(s)+\sum_{\mu=1}^{m / 2}\left(C_{2 \mu} x\right)(s) h^{2 \mu+1}+O\left(h^{m+2}\right), \quad s \in[a, b],
\end{aligned}
$$

where

$$
\begin{aligned}
\left(T_{n} x\right)(s)= & h \sum_{\substack{j \neq 0, a<s+j h \leq b\\
}} k(s, s+j h) x(x+j h)+H_{1}(s, s) x(s) h \log \left(\frac{h}{2 \pi}\right) \\
& +h H_{2}(s, s) x(s),
\end{aligned}
$$

is a modified Nyström scheme proposed in Xu and Zhao (1996) and

$$
C_{2 \mu} x(s)=\left.2 \frac{\zeta^{\prime}(-2 \mu)}{(2 \mu) !}\left(\frac{\partial^{2 \mu}}{\partial t^{2 \mu}} H_{1}(s, t) x(t)\right)\right|_{t=s} \in C^{m+2-2 \mu}(\mathbb{R})
$$

Assume that, for $i=1, \ldots, m,\left.\left(\frac{\partial^{i}}{\partial t^{i}} H_{1}(s, t)\right)\right|_{t=s}$ is periodic with period $Q$. If $x \in C_{Q}^{m+2}(\mathbb{R})$, then

$$
C_{2 \mu} x \in C_{Q}^{m+2-2 \mu}(\mathbb{R}) .
$$

This fact is used in the proof of the Theorem on asymptotic expansion of approximate solution of an operator equation in $\mathrm{Xu}$ and Zhao. Consider the following operator equation

$$
u-T u=f .
$$

Assume that 1 belongs to the resolvent set of $T$. Replacing $T$ by $T_{n}$ and $u$ by $u_{n}$ in equation (7), we obtain 


$$
u_{n}-T_{n} u_{n}=f \text {. }
$$

Since 1 belongs to the resolvent set of $T$ and $T_{n}$ is a collectively compact family of operators converging to $T$ pointwise, for $n$ large enough, equation (8) has a unique solution. The following asymptotic expansion for the approximate solution $u_{n}$ has been proved (see Theorem 2.3; Xu and Zhao, 1996). In what follows, the notation $\lfloor q\rfloor$, denotes the largest integer not greater than $q$.

Theorem 2.2: Let $T$ be an integral operator with the kernel of the class defined in Section 1. Suppose that for $i=1, \ldots, m,\left.\left(\frac{\partial^{i}}{\partial t^{i}} H_{1}(s, t)\right)\right|_{t=s}$ is a periodic function with period $Q$. Then for $s \in[a, b]$, the following asymptotic expansion holds:

$$
\left[\left(I-T_{n}\right)^{-1} f\right](s)=\left[(I-T)^{-1} f\right](s)-v_{3}(s) h^{3}-\sum_{q=5}^{m+1} v_{q}(s) h^{q}+O\left(h^{m+2}\right),
$$

where $v_{q} \in C_{Q}^{(m+2-2\lfloor q / 2\rfloor)}(\mathbb{R})$ are as follows:

$$
\begin{aligned}
& {\left[(I-T) v_{2 q+1}\right]=\left(C_{2 q}\left[(I-T)^{-1} f\right]\right), \quad q=1,2,} \\
& {\left[(I-T) v_{2 q+1}\right]=\left(C_{2 q}\left[(I-T)^{-1} f\right]\right)-\sum_{\mu=2}^{q-1}\left(C_{2 q-2 \mu} \nu_{2 \mu}\right), \quad q=3, \ldots, m / 2}
\end{aligned}
$$

and

$$
v_{4}=0,\left[(I-T) v_{2 q}\right]=-\sum_{\mu=1}^{q-2}\left(C_{2 q-2-2 \mu} \nu_{2 \mu+1}\right), \quad q=3, \ldots, m / 2
$$

Consider the modified Nyström operator $T_{n}$ defined by equation (5). For computing the solutions of

$$
T_{n} \varphi_{n}=\lambda_{n} \varphi_{n}, \quad 0 \neq \lambda_{n} \in \mathbb{C}, \varphi_{n} \in C_{Q}[a, b],
$$

evaluate the above equation at $t_{i}=a+(i-1) h, i=1,2, \ldots, n+1$. Using the periodicity of $k$ and $\varphi_{n}$, we obtain the following matrix eigenvalue problem:

$$
\begin{aligned}
& h \sum_{j \neq i, j=1}^{n+1} k\left(t_{i}, t_{j}\right) \varphi_{n}\left(t_{j}\right)+H_{1}\left(t_{i}, t_{i}\right) \varphi_{n}\left(t_{i}\right) h \log \left(\frac{h}{2 \pi}\right)+h H_{2}\left(t_{i}, t_{i}\right) \varphi_{n}\left(t_{i}\right) \\
& \quad=\lambda_{n} \varphi_{n}\left(t_{i}\right), \quad i=1, \ldots, n+1 .
\end{aligned}
$$

\section{Main results}

Let $\lambda \neq 0$ be a simple eigenvalue of $T$. Let $\varepsilon$ be such that $0<\varepsilon<\operatorname{dist}(\lambda, \operatorname{sp}(T) \backslash\{\lambda\})$ and $\Gamma$ be a positively oriented circle with centre $\lambda$ and radius $\varepsilon$. Then $\Gamma \subseteq \rho(T)$, 


$$
P=-\frac{1}{2 \pi i} \int_{\Gamma}(T-z I)^{-1} d z
$$

is the spectral projection associated with $T$ and $\lambda$ and its rank is 1 .

Let $T_{n}$ be the modified Nyström approximation of $T$ given by equation (5). Since $T_{n}$ is a collectively compact family of operators converging to $T$ pointwise, (see $\mathrm{Xu}$ and $\mathrm{Zhao}$, 1996) it follows that, for all $n$ large enough, $\Gamma \subset \rho\left(T_{n}\right)$. Let

$$
P_{n}=-\frac{1}{2 \pi i} \int_{\Gamma}\left(T_{n}-z I\right)^{-1} d z
$$

be the spectral projection associated with $T_{n}$ and $\Gamma$. Then the rank of $P_{n}=1$ and hence the spectrum of $T_{n}$ inside $\Gamma$ consists of a simple eigenvalue, say $\lambda_{n}$ (see Ahues et al., 2001).

Let $\varphi$ be an eigenvector of $T$ associated with $\lambda$ and normalised by $\int_{a}^{b}|\varphi(s)|^{2} d s=1$. Recall that $\varphi \in C_{Q}^{m+2}(\mathbb{R})$. Let $\varphi_{n}=P_{n} \varphi$. Then for all $n$ large enough, $0 \neq \varphi_{n}$ is an eigenvector associated with $T_{n}$ and $\lambda_{n}$. Using Theorem 2.2, we obtain the asymptotic expansion for $P_{n} \varphi$.

Proposition 3.1: Let $T$ be an integral operator with the kernel defined by equation (2). Suppose that for $i=1, \ldots, m,\left.\left(\frac{\partial^{i}}{\partial t^{i}} H_{1}(s, t)\right)\right|_{t=s}$ is a periodic function of period $Q$. Then for $s \in[a, b]$, we have

$$
\varphi_{n}(s)=\varphi(s)-\chi_{3}(s) h^{3}-\sum_{q=5}^{m+1} \chi_{q}(s) h^{q}+O\left(h^{m+2}\right),
$$

where

$$
\chi_{q}(s)=-\frac{1}{2 \pi i} \int_{\Gamma} v_{q, z}(s) d z,
$$

and

$$
\begin{aligned}
& {\left[(T-z I) v_{2 q+1, z}\right]=-\left(C_{2 q}\left[(T-z I)^{-1} \varphi\right]\right), \quad q=1,2,} \\
& {\left[(T-z I) v_{2 q+1, z}\right]=-\left(C_{2 q}\left[(T-z I)^{-1} \varphi\right]\right)+\sum_{\mu=2}^{q-1}\left(C_{2 q-2 \mu} v_{2 \mu, z}\right), \quad q=3, \ldots, m / 2}
\end{aligned}
$$

and

$$
v_{4, z}=0,\left[(T-z I) v_{2 q, z}\right]=\sum_{\mu=1}^{q-2}\left(C_{2 q-2-2 \mu} \nu_{2 \mu+1, z}\right), q=3, \ldots, m / 2 .
$$

Proof: From equation (4), we have

$$
(T x)(s)=\left(T_{n} x\right)(s)+\sum_{\mu=1}^{m / 2}\left(C_{2 \mu} x\right)(s) h^{2 \mu+1}+O\left(h^{m+2}\right), \quad s \in[a, b] .
$$


For $z \in \rho(T)$, we have

$$
\left[\left(T_{n}-z I\right) x\right](s)=[(T-z I) x](s)-\sum_{\mu=1}^{m / 2}\left(C_{2 \mu} x\right)(s) h^{2 \mu+1}+O\left(h^{m+2}\right), \quad s \in[a, b] .
$$

Now using Theorem 2.2, for all $x \in C_{Q}^{m+2}(\mathbb{R})$ and $s \in[a, b]$, we obtain

$$
\left[\left(T_{n}-z I\right)^{-1} x\right](s)=\left[(T-z I)^{-1} x\right](s)-v_{3, z}(s) h^{3}-\sum_{q=5}^{m+1} v_{q, z}(s) h^{q}+O\left(h^{m+2}\right),
$$

where

$$
\begin{aligned}
& {\left[(T-z I) v_{2 q+1, z}\right]=-\left(C_{2 q}\left[(T-z I)^{-1} x\right]\right), \quad q=1,2,} \\
& {\left[(T-z I) v_{2 q+1, z}\right]=-\left(C_{2 q}\left[(T-z I)^{-1} x\right]\right)+\sum_{\mu=2}^{q-1}\left(C_{2 q-2 \mu} v_{2 \mu, z}\right), \quad q=3, \ldots, m / 2}
\end{aligned}
$$

and

$$
\nu_{4, z}=0,\left[(T-z I) \nu_{2 q, z}\right]=\sum_{\mu=1}^{q-2}\left(C_{2 q-2-2 \mu} \nu_{2 \mu+1, z}\right), q=3, \ldots, m / 2 .
$$

Note that $v_{3, z}, v_{q, z}$ depends upon $\mathrm{z}$ and belong to $C_{Q}^{(m+2-2\lfloor q / 2\rfloor)}(\mathbb{R})$. Integrating along the curve $\Gamma$, we get

$$
\begin{aligned}
-\frac{1}{2 \pi i} \int_{\Gamma}\left[\left(T_{n}-z I\right)^{-1} x\right](s) d z= & -\frac{1}{2 \pi i} \int_{\Gamma}\left[(T-z I)^{-1} x\right](s) d z \\
& -\left(-\frac{1}{2 \pi i} \int_{\Gamma} v_{3, z}(s) d z\right) h^{3} \\
& -\sum_{q=5}^{m+1}\left(-\frac{1}{2 \pi i} \int_{\Gamma} v_{q, z}(s) d z\right) h^{q}+O\left(h^{m+2}\right)
\end{aligned}
$$

Define

$$
\chi_{q}(s)=-\frac{1}{2 \pi i} \int_{\Gamma} v_{q, z}(s) d z, \quad s \in[a, b]
$$

for $q=3,5, \ldots, m+1$, and we have

$$
P_{n} x(s)=P x(s)-\chi_{3}(s) h^{3}-\sum_{q=5}^{m+1} \chi_{q}(s) h^{q}+O\left(h^{m+2}\right), \quad s \in[a, b] .
$$

Substituting $x=\varphi$ in the above equation and noting that $P \varphi=\varphi, P_{n} \varphi=\varphi_{n}$ we obtain equation (11).

We now prove the following proposition. 
Proposition 3.2: Let the conditions of Proposition 3.1 hold. Then for $n$ large enough and $s \in[a, b]$, the following asymptotic expansion holds:

$$
\begin{aligned}
\left(T_{n} \varphi_{n}\right)(s)= & (T \varphi)(s)-\sum_{\mu=1}^{m / 2}\left(C_{2 \mu} \varphi\right)(s) h^{2 \mu+1}-\sum_{q=3, q \neq 4}^{m+1} T \chi_{q}(s) h^{q} \\
& +\sum_{q=3, q \neq 4}^{m-1} \sum_{\mu=1}^{(m / 2)-\lfloor q / 2\rfloor}\left(c_{2 \mu} \chi_{q}\right)(s) h^{2 \mu+1+q}+O\left(h^{m+2}\right),
\end{aligned}
$$

where $C_{2 \mu} x$ is defined in equation (6).

Proof: For $s \in[a, b]$ and $a<s+j h \leq b$, from equation (11) we have

$$
\varphi_{n}(s)=\varphi(s)-\chi_{3}(s) h^{3}-\sum_{q=5}^{m+1} \chi_{q}(s) h^{q}+O\left(h^{m+2}\right)
$$

and

$$
\varphi_{n}(s+j h)=\varphi(s+j h)-\chi_{3}(s+j h) h^{3}-\sum_{q=5}^{m+1} \chi_{q}(s+j h) h^{q}+O\left(h^{m+2}\right) .
$$

For $s \in[a, b]$

$$
\begin{aligned}
\left(T_{n} \varphi_{n}\right)(s)= & h \sum_{j \neq 0, a<s+j h \leq b} k(s, s+j h) \varphi_{n}(s+j h) \\
& +H_{1}(s, s) \varphi_{n}(s) h \log \left(\frac{h}{2 \pi}\right)+h H_{2}(s, s) \varphi_{n}(s) .
\end{aligned}
$$

Using the asymptotic expansions for $\varphi_{n}(s)$ and $\varphi_{n}(s+j h)$ in the above equation, we get

$$
\begin{aligned}
\left(T_{n} \varphi_{n}\right)(s)= & h \sum_{j \neq 0, a<s+j h \leq b} k(s, s+j h)\left(\varphi(s+j h)-\chi_{3}(s+j h) h^{3}-\sum_{q=5}^{m+1} \chi_{q}(s+j h) h^{q}\right) \\
& +H_{1}(s,)\left(\varphi(s)-\chi_{3}(s) h^{3}-\sum_{q=5}^{m+1} \chi_{q}(s) h^{q}\right) h \log \left(\frac{h}{2 \pi}\right) \\
& +h H_{2}(s, s)\left(\varphi(s)-\chi_{3}(s) h^{3}-\sum_{q=5}^{m+1} \chi_{q}(s) h^{q}\right)+O\left(h^{m+2}\right), \quad s \in[a, b] .
\end{aligned}
$$

On arranging the terms, for $s \in[a, b]$, we get 


$$
\begin{aligned}
\left(T_{n} \varphi_{n}\right)(s)= & h \sum_{j \neq 0, a<s+j h \leq b} k(s, s+j h) \varphi(s+j h) \\
& \left.+h\left(H_{1}(s, s) \log \left(\frac{h}{2 \pi}\right)+H_{2}(s, s)\right) \varphi(s)\right) \\
& -\left(h \sum_{j \neq 0, a<s+j h \leq b} k(s, s+j h) \chi_{3}(s+j h)\right. \\
& \left.+h\left(H_{1}(s, s) \log \left(\frac{h}{2 \pi}\right)+H_{2}(s, s)\right) \chi_{3}(s)\right) h^{3} \\
& -\sum_{q=5}^{m+1}\left(h \sum_{j \neq 0, a<s+j h \leq b} k(s, s+j h) \chi_{q}(s+j h)\right. \\
& \left.+h\left(H_{1}(s, s) \log \left(\frac{h}{2 \pi}\right)+H_{2}(s, s)\right) \chi_{q}(s)\right) h^{q} \\
& +O\left(h^{m+2}\right), \quad s \in[a, b] .
\end{aligned}
$$

Thus

$$
\left(T_{n} \varphi_{n}\right)(s)=T_{n} \varphi(s)-T_{n} \chi_{3}(s) h^{3}-\sum_{q=5}^{m+1} T_{n} \chi_{q}(s) h^{q}+O\left(h^{m+2}\right), \quad s \in[a, b] .
$$

For $q=3,5, \ldots, m+1, \chi_{q} \in C_{Q}^{(m+2-2\lfloor q / 2\rfloor)}(\mathbb{R})$ and $\varphi \in C_{Q}^{m+2}(\mathbb{R})$. Using equation (4), for $s \in[a, b]$, we have

$$
\begin{aligned}
& \left.\left(T \chi_{q}\right)(s)=\left(T_{n} \chi_{q}\right)(s)+\sum_{\mu=1}^{(m / 2)-\lfloor q / 2\rfloor}\left(C_{2 \mu} \chi_{q}\right)(s) h^{2 \mu+1}+O\left(h^{(m+2-2\lfloor q / 2\rfloor}\right)\right), \\
& q=3,5,6 \ldots, m-1,
\end{aligned}
$$

and

$$
(T \varphi)(s)=\left(T_{n} \varphi\right)(s)+\sum_{\mu=1}^{m / 2}\left(C_{2 \mu} \varphi\right)(s) h^{2 \mu+1}+O\left(h^{m+2}\right) .
$$

Note that for $s \in[a, b]$,

$$
\left(T \chi_{m}\right)(s)=\left(T_{n} \chi_{m}\right)(s)+O\left(h^{2}\right) \text { and }\left(T \chi_{m+1}\right)(s)=\left(T_{n} \chi_{m+1}\right)(s)+O\left(h^{2}\right) .
$$

Substituting equations (17) and (18) and the above expansions in equation (16), for $s \in[a, b]$ we get 


$$
\begin{aligned}
\left(T_{n} \varphi_{n}\right)(s)= & (T \varphi)(s)-\sum_{\mu=1}^{m / 2} C_{2 \mu} \varphi(s) h^{2 \mu+1}-\left\{T \chi_{3}(s)-\sum_{\mu=1}^{(m / 2)-\lfloor 3 / 2\rfloor} C_{2 \mu} \chi_{3}(s) h^{2 \mu+1}\right\} h^{3} \\
& -\sum_{q=5}^{m-1}\left\{T \chi_{q}(s)-\sum_{\mu=1}^{(m / 2)-\lfloor q / 2\rfloor} C_{2 \mu} \chi_{q}(s) h^{2 \mu+1}\right\} h^{q} \\
& -T \chi_{m}(s) h^{m}-T \chi_{m+1}(s) h^{m+1}+O\left(h^{m+2}\right) .
\end{aligned}
$$

This completes the proof.

Using the asymptotic expansion of $\varphi_{n}(s)$ and $\bar{\varphi}_{n}(s)$, we obtain the following proposition.

Proposition 3.3: Let the conditions of Proposition 3.1 hold. Then we have

$$
\int_{a}^{b}\left|\varphi_{n}(s)\right|^{2} d s=\int_{a}^{b}|\varphi(s)|^{2} d s+\alpha_{3} h^{3}+\sum_{p=5}^{m+1} \alpha_{p} h^{p}+O\left(h^{m+2}\right)
$$

where

$$
\begin{aligned}
& \alpha_{p}=-\int_{a}^{b}\left(\varphi(s) \bar{\chi}_{p}(s)+\bar{\varphi}(s) \chi_{p}(s)\right) d s, \quad p=3,5,7 \\
& \alpha_{6}=\int_{a}^{b}\left(-\varphi(s) \bar{\chi}_{6}(s)-\bar{\varphi}(s) \chi_{6}(s)+\chi_{3}(s) \bar{\chi}_{3}(s)\right) d s, \\
& \alpha_{p}=\int_{a}^{b}\left(-\varphi(s) \bar{\chi}_{p}(s)-\bar{\varphi}(s) \chi_{p}(s)+\sum_{j=3}^{p-3} \chi_{j}(s) \bar{\chi}_{p-j}(s)\right) d s, \quad p \geq 8 .
\end{aligned}
$$

Proof: From Proposition 3.1, we have

$$
\varphi_{n}(s)=\varphi(s)-\chi_{3}(s) h^{3}-\sum_{q=5}^{m+1} \chi_{q}(s) h^{q}+O\left(h^{m+2}\right), \quad s \in[a, b] .
$$

Taking the complex conjugate value of the above equation, we obtain

$$
\bar{\varphi}_{n}(s)=\bar{\varphi}(s)-\bar{\chi}_{3}(s) h^{3}-\sum_{q=5}^{m+1} \bar{\chi}_{q}(s) h^{q}+O\left(h^{m+2}\right), \quad s \in[a, b] .
$$

Multiplying the above equations memberwise, we get

$$
\begin{aligned}
\varphi_{n}(s) \bar{\varphi}_{n}(s)= & \varphi(s) \bar{\varphi}(s) \\
& -\sum_{q=3, q \neq 4}^{m+1}\left(\varphi(s) \bar{\chi}_{q}(s)+\bar{\varphi}(s) \chi_{q}(s)\right) h^{q} \\
& +\sum_{q=3, q \neq 4}^{m+1} \sum_{r=3, r \neq 4}^{m+1} \chi_{q}(s) \bar{\chi}_{r}(s) h^{q+r}+O\left(h^{m+2}\right), \quad s \in[a, b] .
\end{aligned}
$$

Integrating the above equation from $a$ to $b$ we get equation (19).

We now prove the main result concerning asymptotic expansion of $\lambda_{n}$. 
Theorem 3.4: Let the conditions of Proposition 3.1 hold. Let $\lambda$ be a non-zero simple eigenvalue of $T$. Then for $n$ large enough, there exists $\beta_{p}$ independent of $h$ such that the following asymptotic expansion holds:

$$
\lambda_{n}-\lambda=\beta_{3} h^{3}+\sum_{p=5}^{m+1} \beta_{p} h^{p}+O\left(h^{m+2}\right) .
$$

Proof: From equation (13), we have

$$
\begin{aligned}
\left(T_{n} \varphi_{n}\right)(s)= & (T \varphi)(s)-\sum_{\mu=1}^{m / 2}\left(C_{2 \mu} \varphi\right)(s) h^{2 \mu+1}-\sum_{q=3, q \neq 4}^{m+1}\left(T \chi_{q}\right)(s) h^{q} \\
& +\sum_{q=3, q \neq 4}^{m-1} \sum_{\mu=1}^{(m / 2)-\lfloor q / 2\rfloor}\left(C_{2 \mu} \chi_{q}\right)(s) h^{2 \mu+1+q}+O\left(h^{m+2}\right), \quad s \in[a, b] .
\end{aligned}
$$

Since $T \varphi=\lambda \varphi$ and $T_{n} \varphi_{n}=\lambda_{n} \varphi_{n}$, it follows that

$$
\begin{aligned}
\lambda_{n} \varphi_{n}(s)= & \lambda \varphi(s)-\sum_{\mu-1}^{m / 2}\left(C_{2 \mu} \varphi\right)(s) h^{2 \mu+1}-\sum_{q=3, q \neq 4}^{m+1}\left(T \chi_{q}\right)(s) h^{q} \\
& +\sum_{q=3, q \neq 4}^{m-1} \sum_{\mu=1}^{m / 2-\lfloor q / 2\rfloor}\left(C_{2 \mu} \chi_{q}\right)(s) h^{2 \mu+1+q}+O\left(h^{m+2}\right), \quad s \in[a, b] .
\end{aligned}
$$

From equation (11) of Proposition 3.1 we have

$$
\varphi(s)=\varphi_{n}(s)+\chi_{3}(s) h^{3}+\sum_{q=5}^{m+1} \chi_{q}(s) h^{q}+O\left(h^{m+2}\right), \quad s \in[a, b] .
$$

Thus

$$
\begin{aligned}
\left(\lambda_{n}-\lambda\right) \varphi_{n}(s)= & -\sum_{\mu=1}^{m / 2} C_{2 \mu} \varphi(s) h^{2 \mu+1}+\sum_{q=3, q \neq 4}^{m+1}\left\{\lambda \chi_{q}(s)-\left(T \chi_{q}\right)(s)\right\} h^{q} \\
& +\sum_{q=3, q \neq 4}^{m-1} \sum_{\mu=1}^{(m / 2)-\lfloor q / 2\rfloor}\left(C_{2 \mu} \chi_{q}\right)(s) h^{2 \mu+1+q}+O\left(h^{m+2}\right), \quad s \in[a, b] .
\end{aligned}
$$

Multiplying the above equation by $\bar{\varphi}_{n}(s), s \in[a, b]$, we obtain

$$
\begin{aligned}
\left(\lambda_{n}-\lambda\right) \varphi_{n}(s) \bar{\varphi}_{n}(s)= & -\sum_{\mu=1}^{m / 2} \bar{\varphi}(s)\left(C_{2 \mu} \varphi\right)(s) h^{2 \mu+1} \\
& +\sum_{q=3, q \neq 4}^{m+1} \bar{\varphi}(s)\left\{\lambda \chi_{q}(s)-\left(T \chi_{q}\right)(s)\right\} h^{q} \\
& +\sum_{q=3, q \neq 4}^{m-1} \sum_{\mu=1}^{m / 2-\lfloor q / 2\rfloor} \bar{\varphi}(s)\left(C_{2 \mu} \chi_{q}\right)(s) h^{2 \mu+1+q}
\end{aligned}
$$




$$
\begin{aligned}
& +\sum_{\mu=1}^{m / 2} \sum_{r=3, r \neq 4}^{m+1} \bar{\chi}_{r}(s)\left(C_{2 \mu} \chi_{q}\right)(s) h^{2 \mu+1+r} \\
& -\sum_{q=3, q \neq 4}^{m+1} \sum_{r=3, r \neq 4}^{m+1} \bar{\chi}_{r}(s)\left\{\lambda \chi_{q}(s)-\left(T \chi_{q}\right)(s)\right\} h^{q+r} \\
& -\sum_{q=3, q \neq 4}^{m-1} \sum_{\mu=1}^{m / 2-\lfloor q / 2\rfloor} \sum_{r=3, r \neq 4}^{m+1} \bar{\chi}_{r}(s)\left(C_{2 \mu} \chi_{q}\right)(s) h^{2 \mu+1+q+r} \\
& +O\left(h^{(m+2)}\right) .
\end{aligned}
$$

It can be seen that the above expression has terms of order $h^{3}, h^{5}, h^{6}, h^{7}, \ldots$ It is possible to write the above equation as

$$
\left(\lambda_{n}-\lambda\right) \varphi_{n}(s) \bar{\varphi}_{n}(s)=\omega_{3}(s) h^{3}+\sum_{p=5}^{m+1} \omega_{p}(s) h^{p}+O\left(h^{(m+2)}\right), \quad s \in[a, b] .
$$

A first few terms $\omega_{p}$ are of the form

$$
\begin{aligned}
& \omega_{3}=-\bar{\varphi}\left(C_{2} \varphi\right)+\bar{\varphi}\left\{\lambda \chi_{3}-\left(T \chi_{3}\right)\right\}, \\
& \omega_{5}=-\bar{\varphi}\left(C_{4} \varphi\right)+\bar{\varphi}\left\{\lambda \chi_{5}-\left(T \chi_{5}\right)\right\}, \\
& \omega_{6}=\bar{\varphi}\left(C_{2} \chi_{3}\right)+\bar{\chi}_{3}\left(C_{2} \varphi\right)+\bar{\varphi}\left\{\lambda \chi_{6}-\left(T \chi_{6}\right)\right\}+\bar{\chi}_{3}\left\{\lambda \chi_{3}-T \chi_{3}\right\}, \\
& \omega_{7}=-\bar{\varphi}\left(C_{6} \varphi\right)+\bar{\varphi}\left\{\lambda \chi_{7}-\left(T \chi_{7}\right)\right\}
\end{aligned}
$$

and

$$
\begin{aligned}
\omega_{8}= & \bar{\varphi}\left\{\lambda \chi_{8}-\left(T \chi_{8}\right)\right\}+\bar{\varphi}\left(C_{4} \chi_{3}\right)+\bar{\varphi}\left(C_{2} \chi_{5}\right) \\
& +\bar{\chi}_{3}\left(C_{4} \varphi\right)+\bar{\chi}_{5}\left(C_{2} \varphi\right)+\bar{\chi}_{3}\left\{\lambda \chi_{5}-\left(T \chi_{5}\right)\right\}+\bar{\chi}_{5}\left\{\lambda \chi_{3}-\left(T \chi_{3}\right)\right\} .
\end{aligned}
$$

Integrating equation (32) with respect to $s$ and using equation (9), we obtain

$$
\begin{aligned}
\left(\lambda_{n}-\lambda\right) & =\frac{\int_{a}^{b} \omega_{3}(s) d s h^{3}+\sum_{p=5}^{m+1} \int_{a}^{b} \omega_{p}(s) d s h^{p}+O\left(h^{(m+2)}\right)}{\int_{a}^{b}|\varphi(s)|^{2} d s+\alpha_{3} h^{3}+\sum_{p=5}^{m+1} \alpha_{p} h^{p}+O\left(h^{m+2}\right)} \\
& =\frac{\int_{a}^{b} \omega_{3}(s) d s h^{3}+\sum_{p=5}^{m+1} \int_{a}^{b} \omega_{p}(s) d s h^{p}+O\left(h^{(m+2)}\right)}{1+\alpha_{3} h^{3}+\sum_{p=5}^{m+1} \alpha_{p} h^{p}+O\left(h^{m+2}\right)} \\
= & \beta_{3} h^{3}+\sum_{p=5}^{m+1} \beta_{p} h^{p}+O\left(h^{m+2}\right)
\end{aligned}
$$


where $\beta_{p}$ are recursively chosen as

$$
\begin{aligned}
& \beta_{3}=\int_{a}^{b} \omega_{3}(s) d s, \beta_{5}=\int_{a}^{b} \omega_{5}(s) d s, \beta_{7}=\int_{a}^{b} \omega_{7}(s) d s, \\
& \beta_{6}+\alpha_{3} \beta_{3}=\int_{a}^{b} \omega_{6}(s) d s
\end{aligned}
$$

and

$$
\beta_{p}+\sum_{\substack{j=3 \\ j \neq 4, p-4}}^{p-3} \beta_{j} \alpha_{p-j}=\int_{a}^{b} \omega_{p}(s) d s, \quad p>7 .
$$

\section{Extrapolation}

We propose an extrapolation scheme for the eigenvalue and consider a numerical example. Let

$$
\lambda_{n}^{(0)}=\lambda_{n}, \lambda_{n}^{(1)}=\frac{8 \lambda_{2 n}^{(0)}-\lambda_{n}^{(0)}}{7},
$$

and

$$
\lambda_{n}^{(l)}=\frac{2^{l+3} \lambda_{2 n}^{(l-1)}-\lambda_{n}^{(l-1)}}{2^{l+3}-1}, \quad l=2,3, \ldots
$$

Theorem 4.1: Let the conditions of Proposition 3.1 hold. Let $\lambda$ be a simple non-zero eigenvalue of $T$. Then

$$
\lambda_{n}^{(l)}=\lambda+\sum_{j=l+4}^{m+1} v_{j}^{(l)} h^{j}+O\left(h^{m+2}\right), \quad l=1,2, \ldots
$$

where $v_{j}^{(l)}$ are scalars independent of $h$.

\subsection{Numerical example}

Consider

$$
\int_{0}^{2 \pi} k(s, t) \varphi(t) d t=\lambda \varphi(s), \quad 0 \leq s \leq 2 \pi
$$

with

$$
k(s, t)=\log \left(4 \sin \frac{|s-t|}{2}\right), \quad s, t \in[0,2 \pi] .
$$


We approximate the eigenvalue $-\pi$ by $\lambda_{n}^{(l)}$ using the modified trapezoidal rule. The expected orders of convergence are $\alpha_{0}=3, \alpha_{1}=5, \alpha_{2}=6$ and $\alpha_{3}=7$.

Table 1 Modified trapezoidal rule

\begin{tabular}{lcccccccc}
\hline$n$ & $\left|\lambda-\lambda_{n}\right|$ & $\alpha_{0}$ & $\left|\lambda-\lambda_{n}^{(1)}\right|$ & $\alpha_{1}$ & $\left|\lambda-\lambda_{n}^{(2)}\right|$ & $\alpha_{2}$ & $\left|\lambda-\lambda_{n}^{(3)}\right|$ & $\alpha_{3}$ \\
\hline 4 & $1.25 \times 10^{-1}$ & & & & & & & \\
8 & $1.50 \times 10^{-2}$ & 3.06 & $7.37 \times 10^{-4}$ & & & & & \\
16 & $1.85 \times 10^{-3}$ & 3.05 & $2.18 \times 10^{-5}$ & 5.09 & $1.36 \times 10^{-6}$ & & & \\
32 & $2.31 \times 10^{-4}$ & 3.00 & $6.69 \times 10^{-7}$ & 5.02 & $9.99 \times 10^{-9}$ & 7.09 & $1.15 \times 10^{-8}$ & \\
64 & $2.88 \times 10^{-5}$ & 3.00 & $2.08 \times 10^{-8}$ & 5.01 & $7.69 \times 10^{-11}$ & 7.02 & $8.05 \times 10^{-11}$ & 7.16 \\
128 & $3.60 \times 10^{-6}$ & 3.00 & $6.50 \times 10^{-10}$ & 5.00 & $5.99 \times 10^{-13}$ & 7.00 & $6.12 \times 10^{-13}$ & 7.04 \\
\hline
\end{tabular}

The numerical results thus match the theoretical rates of convergence.

\section{Acknowledgements}

The work of the first author was partially supported by CEFIPRA, Project No. 4101-1.

\section{References}

Ahues, M., Largillier, A. and Limaye, B.V. (2001) Spectral Computations for Bounded Operators, Chapman and Hall/CRC, USA.

Baker, C.T.H. (1977) The Numerical Treatment of Integral Equations, Oxford University Press, Oxford.

Kulkarni, R.P. (1997) 'Use of extrapolation for improving the order of convergence of eigenelement approximations', IMA J. Numer. Anal., Vol. 17, No. 2, pp.271-284.

Kulkarni, R.P. and Rane, A.S. (2012) 'Asymptotic expansions for approximate eigenvalues of integral operators with nonsmooth kernels', Numer. Funct. Anal. Optim., Vol. 33, No. 4, pp.415-440.

Richter, G.R. (1976) 'On weakly singular Fredholm integral equations with displacement kernels', J. Math. Anal. Appl., Vol. 55, No. 1, pp.32-42.

Schneider, C. (1979) 'Regularity of the solution to a class of weakly singular Fredholm integral equations of the second kind', Int. Eqs. Op. Thy., Vol. 2, No. 2, pp.62-68.

Sidi, A. (1989) 'Comparison of some numerical quadrature formulas for weakly singular periodic Fredholm integral equations', Computing, Vol. 43, No. 2, pp.159-170.

$\mathrm{Xu}, \mathrm{Y}$. and Zhao, Y. (1996) 'An extrapolation method for a class of boundary integral equations', Math. Comp., Vol. 65, No. 214, pp.587-610. 